(1)

CrossMark

\title{
Needle-based confocal laser endomicroscopy for real-time diagnosing and staging of lung cancer
}

\author{
Lizzy Wijmans ${ }^{1}$, Joey Yared ${ }^{1}$, Daniel M. de Bruin², Sybren L. Meijer³, \\ Paul Baas ${ }^{4}$, Peter I. Bonta ${ }^{1}$ and Jouke T. Annema ${ }^{1}$
}

Affiliations: ${ }^{1}$ Dept of Respiratory Medicine, Amsterdam University Medical Centers, Amsterdam, The Netherlands. ${ }^{2}$ Dept of Biomedical Engineering and Physics, Amsterdam University Medical Centers, Amsterdam, The Netherlands. ${ }^{3}$ Dept of Pathology, Amsterdam University Medical Centers, Amsterdam, The Netherlands. ${ }^{4}$ Dept of Pulmonology, National Cancer Institute AvL - NKI, Amsterdam, The Netherlands.

Correspondence: Jouke T. Annema, Dept of Pulmonology, G5-247, Amsterdam University Medical Centers, University of Amsterdam, Meibergdreef 9, 1105 AZ Amsterdam, P.O. Box 22700, 1100 DE Amsterdam, The Netherlands. E-mail: j.t.annemadamsterdamumc.nl

@ERSpublications

Real-time lung cancer detection by endosonography-guided nCLE was feasible and safe. nCLE lung cancer characteristics were accurately recognised. nCLE might become a useful adjunct to bronchoscopy for real-time identification of lung tumours. http://ow.ly/PE7m $3008 \mathrm{rSn}$

Cite this article as: Wijmans L, Yared J, de Bruin DM, et al. Needle-based confocal laser endomicroscopy for real-time diagnosing and staging of lung cancer. Eur Respir J 2019; 53: 1801520 [https://doi.org/ 10.1183/13993003.01520-2018].

ABSTRACT Diagnosing lung cancer in the absence of endobronchial abnormalities is challenging. Needle-based confocal laser endomicroscopy (nCLE) enables real-time microscopic imaging of cells. We assessed the feasibility and safety of using nCLE for real-time identification of lung cancer.

In patients with suspected or proven lung cancer scheduled for endoscopic ultrasound (EUS), lung tumours and mediastinal lymph nodes were imaged with nCLE before fine-needle aspiration (FNA) was performed. nCLE lung cancer characteristics were identified by comparison with pathology. Multiple blinded raters validated CLE videos of lung tumours and mediastinal nodes twice.

EUS-nCLE-FNA was performed in 22 patients with suspected or proven lung cancer in whom 27 lesions (six tumours, 21 mediastinal nodes) were evaluated without complications. Three nCLE lung cancer criteria (dark enlarged pleomorphic cells, dark clumps and directional streaming) were identified. The accuracy of nCLE imaging for detecting malignancy was $90 \%$ in tumours and $89 \%$ in metastatic lymph nodes. Both inter-observer agreement (mean $\kappa=0.68,95 \%$ CI 0.66-0.70) and intra-observer agreement (mean \pm SD $\kappa=0.70 \pm 0.15$ ) were substantial.

Real-time lung cancer detection by endosonography-guided nCLE was feasible and safe. Lung cancer characteristics were accurately recognised. 


\section{Introduction}

With 2 million incident cases and 1.7 million deaths, lung cancer is amongst the commonest and deadliest cancers worldwide [1]. Establishing a tissue diagnosis is a prerequisite for treatment and prognosis. With the increased use of chest computed tomography (CT) and expected upcoming lung cancer screening, the need for improved bronchoscopic navigation, including tumour identification techniques, is urgent.

Lung cancer diagnosis and staging has been revolutionised in the last decade, primarily with the advent of radial and linear endobronchial ultrasound (EBUS). However, despite the use of fluoroscopy, radial EBUS and electromagnetic navigation as bronchoscopy-guidance tools for peripheral lung lesions, there is room for improvement in order to achieve a high diagnostic yield [2].

To date, a lot of effort has been devoted to the development of guided bronchoscopic techniques to reach peripheral lung lesions [3]. In the future, it would be ideal if bronchoscopic treatment could be performed immediately after localisation of the target lesion by navigational techniques. A key step in this desirable future concept is real-time confirmation of the malignancy status of the nodule.

Confocal laser endomicroscopy (CLE) is a laser-based technique that is executed with a fluorescent dye and enables real-time visualisation of cell shapes, thereby acting as a real-time microscope [4-6]. The value of the "surface-scanning" approach (probe-based CLE (pCLE)), with the use of the safe staining fluorescein, has been studied in lung cancer patients but does not allow the visualisation of individual malignant cells [7-9]. Through recent advances, the imaging probe has become small enough to be advanced through a biopsy needle (needle-based CLE (nCLE)). We recently demonstrated, in a single case of a centrally located pulmonary tumour, that high resolution "through the needle" imaging with nCLE enables real-time visualisation of tumour cells within a lung tumour [10].

Application of the nCLE technique in lung cancer diagnostics has the potential to provide real-time feedback to the endoscopist about the malignancy status of the lesion under investigation. Data concerning the feasibility, characteristics and interpretability of nCLE imaging in lung cancer are currently lacking. We hypothesise that the nCLE technique enables real-time visualisation of malignant cellular structures in pulmonary tumours and metastatic lymph nodes. In addition, this study was designed to assess whether trained observers can interpret the nCLE imaging in lung cancer patients.

\section{Materials and methods}

\section{Study design}

This is an ethically approved single-centre study (ClinicalTrials.gov NCT02689050) conducted between February 2016 and March 2017 in the Academic Medical Center in Amsterdam, The Netherlands. Patients undergoing endoscopic ultrasound (EUS) for the diagnosis and staging of lung cancer were eligible for inclusion. Exclusion criteria were age $<18$ years, pregnancy and a known allergy to fluorescein. Because anaphylactic shock is a rare but potentially life-threatening side effect of the intravenous use of fluorescein [11] and beta-blocker use is known to increase the risk of severe anaphylaxis [12], patients with beta-blocker use $<12 \mathrm{~h}$ prior to the procedure were excluded from study participation. We chose an EUS approach because the confocal probe fitted through a 19-gauge (19G) EUS needle but not a 19G EBUS needle. Written informed consent was obtained from all patients. The final diagnosis (reference standard) was based on cytology results of EUS-fine-needle aspiration (FNA) in cases of a tumour-positive sample or on surgical-pathological staging or clinical and radiological follow-up (CT and/or positron emission tomography (PET) scan) for a minimum of 10 months in cases with no tumour (metastasis) on FNA. nCLE videos were obtained with the Cellvizio system (Mauna Kea Technologies, Paris, France) using the AQ-Flex 19 miniprobe with a diameter of $<1 \mathrm{~mm}$ and a resolution of $3.5 \mu \mathrm{m}$.

In order to identify and validate nCLE criteria for malignant lesions involved in lung cancer, this study consisted of three phases (figure 1). Phase 1 involved identification of the nCLE lung cancer criteria. This was followed by two rating sessions (phases 2 and 3). During the rating sessions, blinded raters scored a set of nCLE videos for the presence of the identified criteria.

The videos used for all three sessions consisted of preselected video sequences (a total of 47 preselected videos of 27 evaluable lesions) in order to make the sessions a feasible duration. For every evaluable nCLE-imaged lesion, at least one video sequence was selected for one of the sets (identification of CLE criteria (seven videos), rater training (six videos) or validation set (34 videos)).

\section{Study procedure}

EUS procedures (Pentax Slim scope, EG-3270UK; Pentax, Tokyo, Japan) were performed by a single endoscopist with the patient under deep or moderate sedation. EUS inspection was performed and the following ultrasound characteristics of the target lesions were registered: shape (flat/oval versus round), size $(<10 \mathrm{~mm}$ versus $>10 \mathrm{~mm}$ ), echogenicity (iso- versus hypo-echoic), borders (vague versus sharp) and central 
Included patients ( $\mathrm{n}=22)$

nCLE-imaged lymph nodes/pulmonary masses $(n=34)$

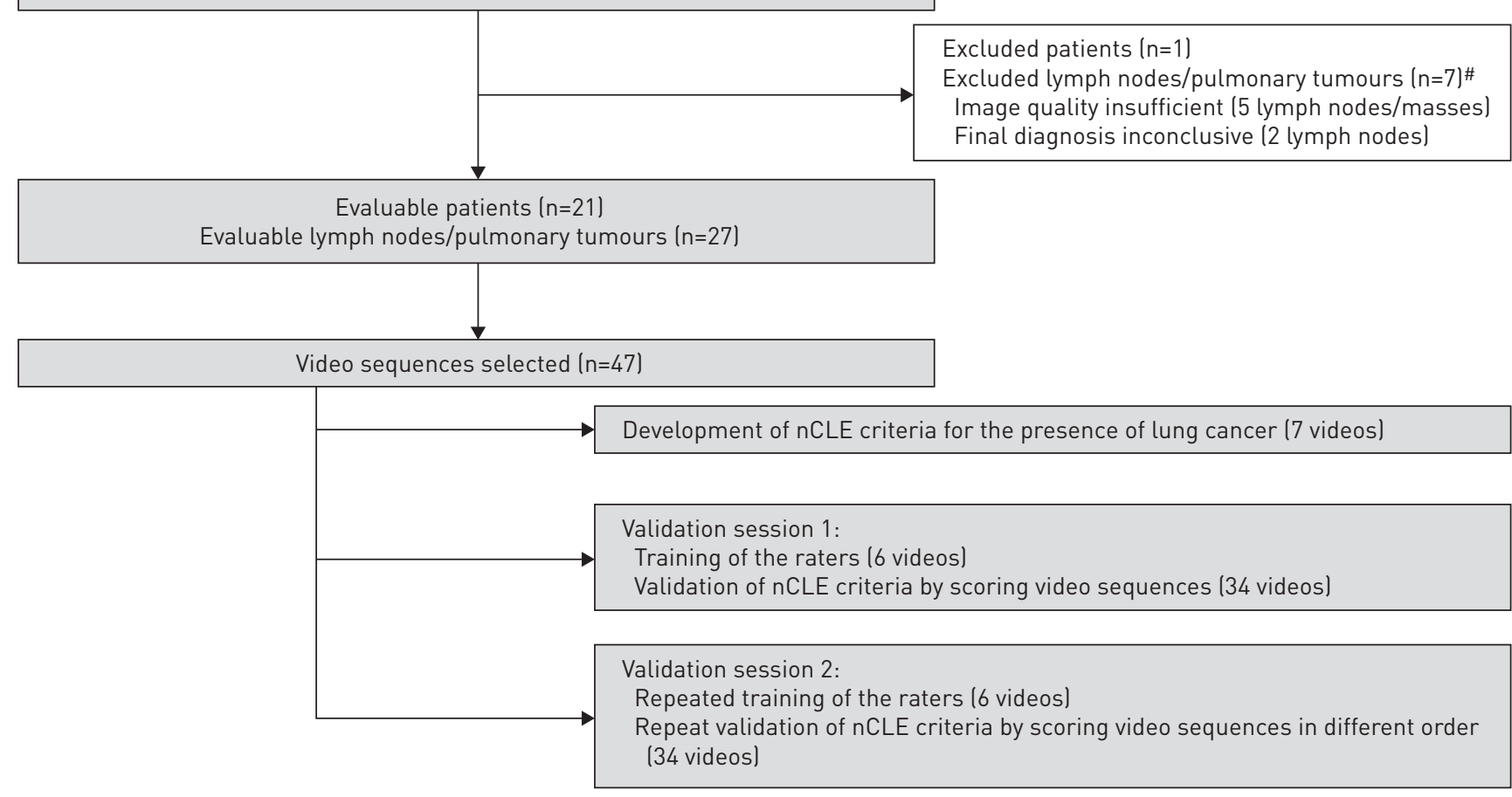

FIGURE 1 Study flow diagram. ": In total, seven needle-based confocal laser endomicroscopy (nCLE)-imaged lesions were excluded from analysis, because of poor image quality (five lymph nodes/pulmonary tumours) or an inconclusive final diagnosis (two lymph nodes). Because several of these mediastinal nodes were assessed in a single patient, this led to the exclusion of only one patient.

hilar sign (presence versus absence). After EUS inspection of the mediastinal nodes and para-oesophageally located lung tumour, $2.5 \mathrm{~mL}$ of $10 \%$ fluorescein-dinatrium solution was injected intravenously. Prior to the procedure, a 19G FNA needle (Cook Medical, Limerick, Ireland) was preloaded with the AQ-Flex 19 miniprobe (Cellvizio). Following target identification, the needle containing the preloaded CLE miniprobe was inserted into the lung tumour or mediastinal lymph node under EUS guidance (figure 2). Subsequently, the nCLE probe was advanced and securely positioned using a locking device, whereafter video sequences from within the tumour/lymph node were recorded while moving the needle and nCLE probe forwards under real-time ultrasound visualisation. After nCLE image acquisition, the nCLE probe was removed, followed by several needle passes in the same plane as the previous nCLE imaging. In a single patient, multiple lesions could be imaged with nCLE and sampled. FNA samples were processed on glass slides, with conventional hematoxylin and eosin staining, and cell blocks were fabricated. Rapid on-site cytological evaluation (ROSE) was available. Patients received antibiotic prophylaxis in cases of suspected necrosis during EUS imaging or ROSE assessment.

\section{Identification of CLE criteria}

Three reviewers (a pathologist, an endoscopist and a researcher) reviewed nCLE videos to assess nCLE criteria for the presence of malignancy. The reviewers compared the nCLE imaging to the corresponding cytopathology and were aware of the final diagnosis.

\section{Training session}

The seven raters were trained for $\sim 15 \mathrm{~min}$ in the newly identified nCLE lung cancer criteria using an independent set of training videos that had not been used in the previous identification session. Training slides with hand-drawn nCLE lung cancer criteria schematics were provided to the raters immediately preceding both validation sessions.

\section{Validation sessions}

Immediately after training, the first validation session was performed to assess the diagnostic yield and the inter-observer agreement (IOA) between the raters for detecting malignancies based on nCLE videos. The raters were blinded to the final diagnosis. A set of 34 selected nCLE videos that had not been used for identifying the criteria or for training were reviewed by seven blinded raters with different backgrounds 

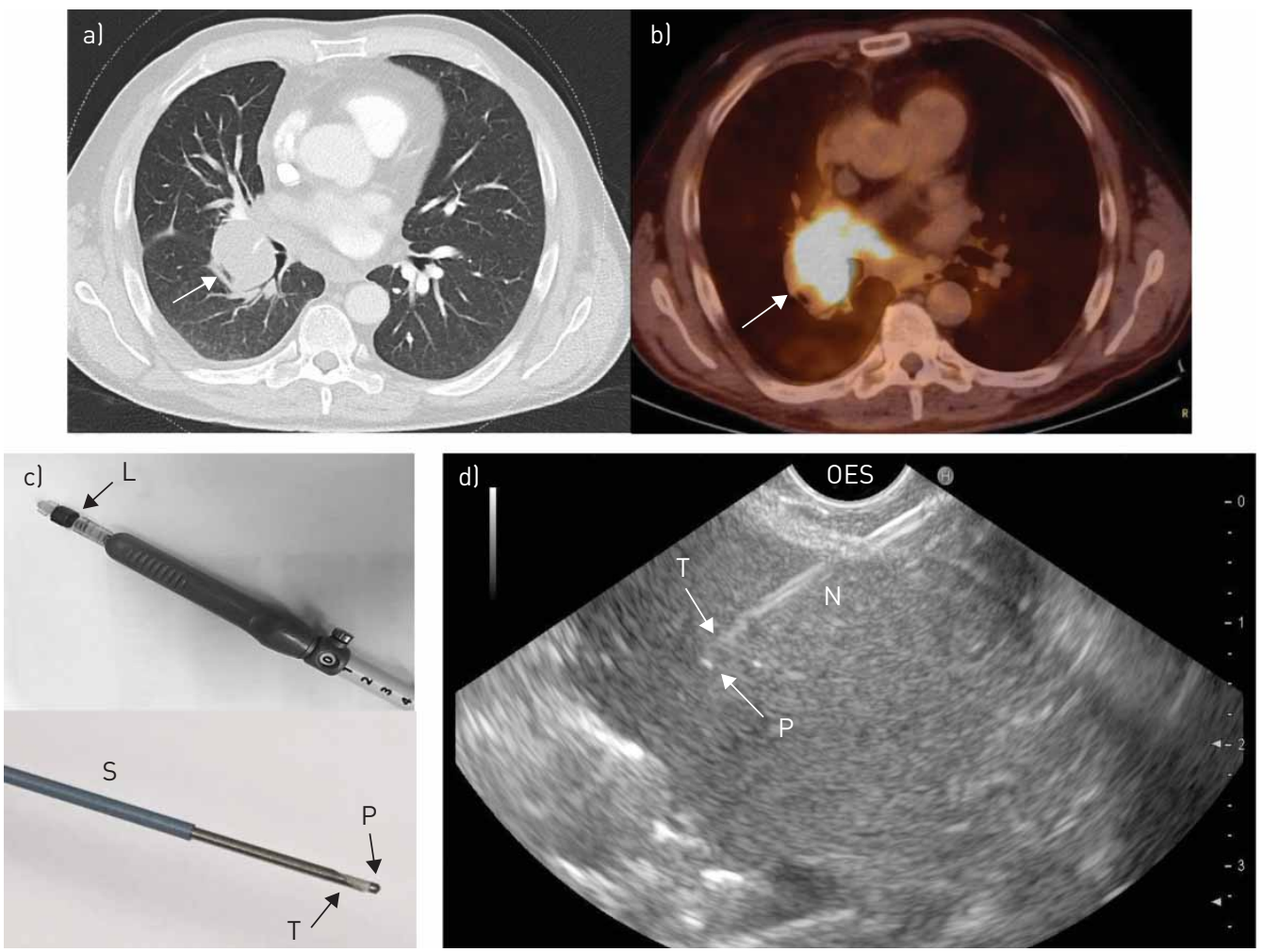

FIGURE 2 a) Chest computed tomography scan with lung tumour (arrow) and b) positron emission tomography-computed tomography scan with intense 2-fluoro-2-deoxy-D-glucose uptake (arrow) by the tumour. c) Preparation of the needle: the Luer lock (L) is adjusted on top of the needle handle of a 19G Cook needle. The needle is covered by a sheath $(S)$ and the $A Q-F l e x$ miniprobe $(P)$ is locked $1 \mathrm{~mm}$ in front of the needle tip (T). d) Real-time endoscopic ultrasound-guided needle-based confocal laser endomicroscopy fine-needle aspiration. The needle $(\mathrm{N})$ is placed in a pulmonary tumour from an oesophageal (OES) approach under real-time ultrasound guidance. The confocal miniprobe (P) is positioned in front of the needle tip (T). Scale bar: $1 \mathrm{~cm}$.

(three endosonographers, two cytology technicians and two CLE experts in the field of urology) who had not been involved in defining the $\mathrm{nCLE}$ criteria for lung cancer. A case report form was scored for the presence of the three criteria for malignancy (yes/no), the final diagnosis (malignant/non-malignant) and the level of confidence (good/moderate/poor).

The second validation session was performed by the same group of raters, after a 2-week wash-out period, using the same set of PowerPoint slides and nCLE videos in a different order (figure 1).

\section{Statistical analysis}

The IOA was calculated using MATLAB and Statistics Toolbox Release 2012b (The MathWorks Inc., Natick, MA, USA). The sensitivity, specificity, positive predictive value, negative predictive value, diagnostic accuracy and intra-observer reliability (IOR) were performed using the standard definitions and software from SPSS statistical package, version 24.0 (IBM Corporation, Somers, NY, USA). The performance of the raters for distinguishing malignancy was calculated using the ratings of 34 videos by seven raters (238 ratings in total) from the first validation session. The IOA was calculated using multi-rater Fleiss's $\kappa$ statistics and the mean IOR by comparing the results of the first and the second validation sessions using Cohen's $\kappa$. The IOA and IOR results were interpreted using the Landis-Koch interpretation system: poor $<0.2$, fair $0.21-0.4$, moderate $0.41-0.6$, substantial $0.61-0.8$ and excellent $0.81-1[13,14]$.

\section{Results}

\section{Patient characteristics}

EUS-nCLE-FNA was performed in 22 patients with suspected or proven lung cancer with an enlarged or PET-positive mediastinal lymph node or a centrally located lung tumour. The assessed malignant lymph nodes featured on average three to five of the registered ultrasound characteristics for malignancy. Demographics and final diagnoses are displayed in table 1. 


\begin{tabular}{lc} 
TABLE 1 Patient characteristics and final diagnosis & Value \\
Variable & 21 \\
\hline Evaluable patients & $67.9 \pm 9.4$ \\
Age years (mean士sD) & 15 \\
Sex & 6 \\
Male & 21 \\
Female & 15 \\
Final diagnosis & 6 \\
NSCLC & 8 \\
$\quad$ Stage I/II & 1 \\
Stage III & 4 \\
Stage IV & 1 \\
SCLC limited disease & 1 \\
Mesothelioma & 15 \\
IgG4-related disease & 1
\end{tabular}

Data are presented as $\mathrm{n}$, unless otherwise stated. NSCLC: nonsmall cell lung cancer; SCLC: small cell lung cancer; Ig: immunoglobulin.

nCLE imaging was obtained from five pulmonary and one mediastinal tumour. Final diagnoses were adenocarcinoma $(n=3)$, squamous cell carcinoma (SCC) $(n=1)$, small cell lung carcinoma (SCLC) $(n=1)$ and malignant mesothelioma $(n=1)$. nCLE imaging of 21 mediastinal lymph nodes was performed. The final diagnoses of 15 malignant lymph nodes were adenocarcinoma $(n=4)$, SCC $(n=4)$, SCLC $(n=6)$ and nonsmall cell lung carcinoma (NSCLC) undifferentiated $(n=1)$; six nodes were reactive.

Of these six lymph nodes, surgical-pathological verification of EUS-FNA findings was available in one patient, confirming the presence of a reactive lymph node. The other five nodes were followed up with CT and/or PET-CT for a mean duration of 10 months (range 9.8-11 months). The sensitivity, specificity, diagnostic accuracy, positive predictive value and negative predictive value of EUS-FNA cytology were $95.2 \%, 100 \%, 96.3 \%, 100 \%$ and $85.7 \%$, respectively.

Five out of 34 lesions were not included in the analysis because of technical issues with nCLE imaging owing to stain on the probe (three lesions imaged during one procedure) and improper contact between the probe and the tissue (two lesions). Two other lesions were excluded from the analysis owing to the absence of a conclusive final diagnosis (figure 1). There were no adverse events related to the nCLE imaging or the fluorescein administration.

\section{CLE-identified criteria}

All CLE-imaged tumour types featured enlarged pleomorphic cells on CLE imaging (figure 3). Additionally, CLE visualised glandular-shaped structures in a pulmonary tumour that correlated to the histology of an adenocarcinoma (figure 4). Necrosis caused a loss of CLE signal in two cases with SCC; however, this sign was not specific for SCC.

In different subtypes of lung tumours (figure 4) and metastatic lymph nodes (figure 5), similar nCLE characteristics for the presence of malignancy were found. Three nCLE characteristics for the detection of malignancy were identified: 1) dark enlarged pleomorphic cells, 2) dark cell clusters consisting of overlapping cell-structures ("dark clumps") and 3) the continuous movement of a part of the cells in one direction ("directional streaming"). An example video with the three identified nCLE characteristics is available in the supplementary material.

In reactive lymph nodes the following structures were identified based on nCLE imaging: capsule and cortex of a lymph node and adipocytes (figure 5). Metastatic lymph nodes also featured these characteristics and additionally showed the presence of at least one of the above-mentioned characteristics for malignancy (indicated in figure 5 by $\mathrm{M}$ ).

\section{Detection of malignancy}

Overall sensitivity and specificity of nCLE imaging for lung cancer malignancy detection for the seven raters was 0.90 (95\% CI 0.94-0.99) and 0.89 (95\% CI 0.73-0.95), respectively (table 2). From the nCLE videos that were rated with "good" $(\mathrm{n}=120,50.4 \%)$ or "moderate" ( $\mathrm{n}=95,39.9 \%)$ confidence, only $5 \%$ and 


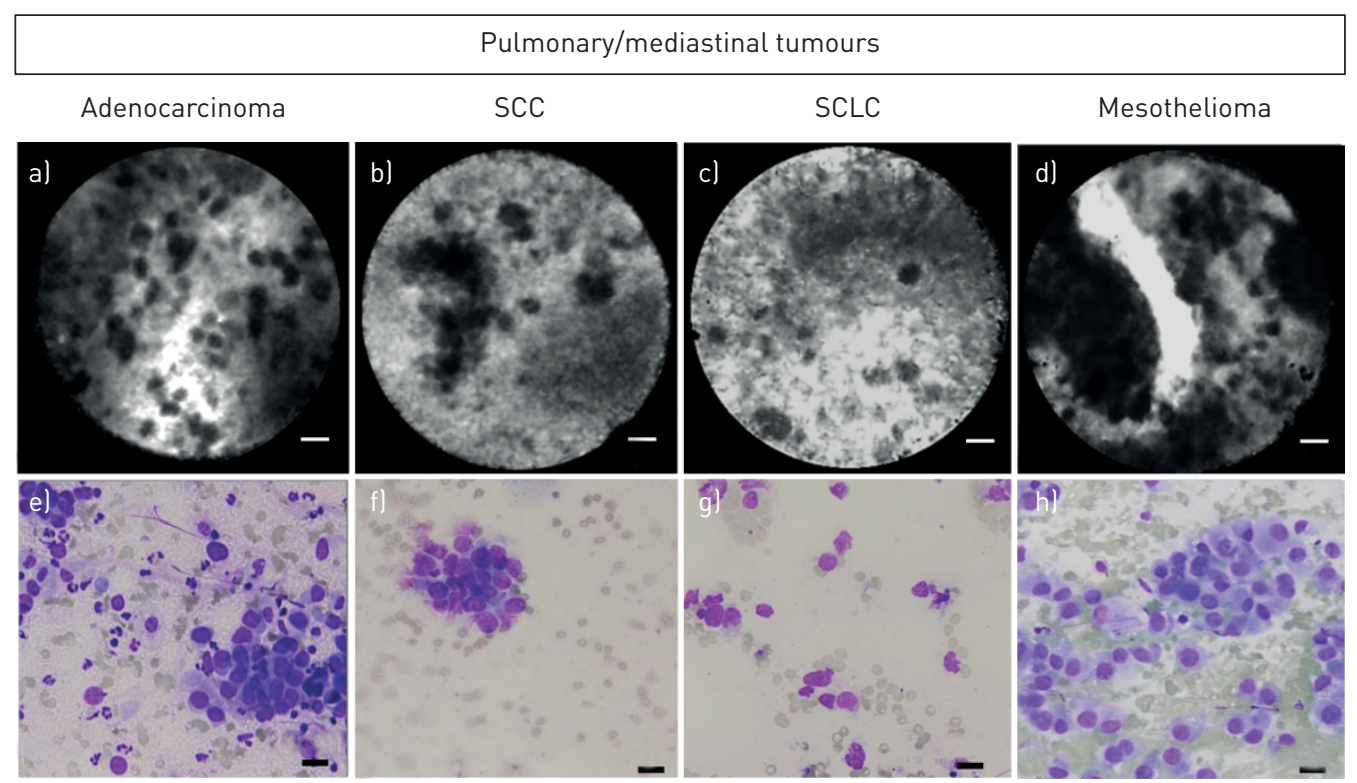

FIGURE 3 a-d) Real-time in vivo needle-based confocal laser endomicroscopy imaging of different pulmonary tumours (adenocarcinoma, squamous cell carcinoma (SCC), small cell lung carcinoma (SCLC) and malignant mesothelioma epithelial type located in the mediastinum) with e-h) corresponding cytology of the fine-needle aspiration. An example video is available as supplementary video. All types of tumours featured the presence of enlarged pleomorphic cell shapes. The bright structure in d is a vessel. Scale bars: $20 \mu \mathrm{m}$.

$10.5 \%$ were rated incorrectly. In sequences that were rated with "poor" confidence ( $\mathrm{n}=23,9.7 \%$ of total), $26.1 \%$ were rated incorrectly (supplementary table).

Inter-observer agreement and intra-observer reliability

The overall agreement for the presence of malignancy in validation session 1 calculated for the seven raters was substantial (IOA $\kappa=0.68,95 \%$ CI $0.66-0.70$ ) (table 3 ).

In terms of the separate criteria, there was moderate agreement (IOA $\kappa=0.48,95 \%$ CI $0.46-0.50$ ) between the raters for the criterion "enlarged cells", which was present in $85 \%$ of movies of malignant lesions. There was fair agreement (IOA $\kappa=0.31,95 \%$ CI 0.29-0.33) for the criterion "dark clumps", which was present in $38 \%$ of movies of malignant lesions. There was substantial agreement (IOA $\kappa=0.62,95 \%$ CI $0.60-0.64$ ) for the criterion "directional streaming", which was present in $12 \%$ of movies of malignant lesions.

The mean \pm SD IOR, calculated by comparing the raters' performances between validation sessions 1 and 2, was "substantial" ( $\kappa=0.70 \pm 0.15)$ for diagnosing malignancy (table 3 ).
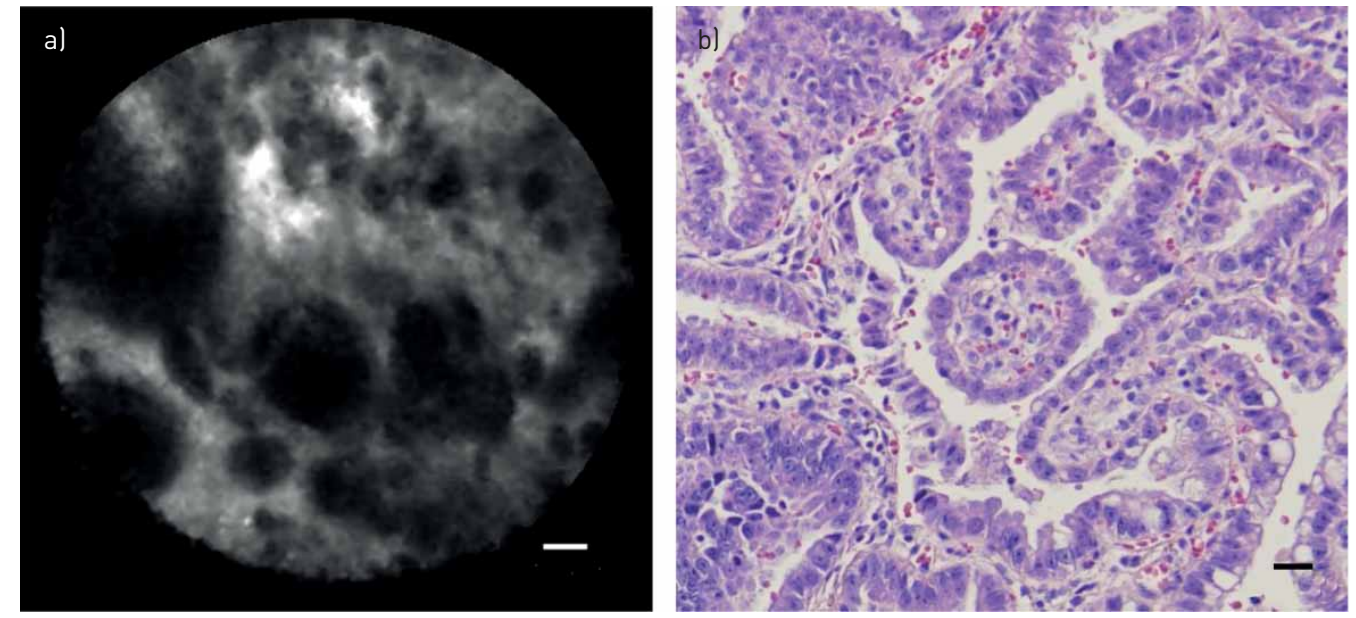

FIGURE 4 a) Ex vivo needle-based confocal laser endomicroscopy image of an adenocarcinoma of the lung with b) corresponding histology with formation of glandular structures. Scale bars: $20 \mu \mathrm{m}$. 


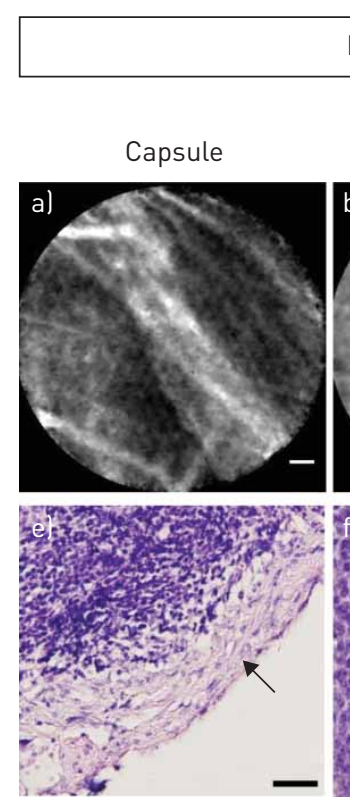

FIGURE 5 a-c) In vivo needle-based confocal laser endomicroscopy (nCLE) imaging of reactive lymph node structures with the e-g) corresponding histology image after surgical excision of the node. a) The dark background with bright fibre structure represents the capsular structures of a lymph node lindicated in e by the arrow). b) Homogenously distributed grey dots visualised in the cortex of a lymph node with homogenously shaped and sized lymphocytes (f). c) Large dark bubbles represent adipocytes (g). d) In vivo nCLE imaging of a metastatic lymph node with a background of cortex-appearance and a cluster of enlarged cells with h) corresponding cytology of the fine-needle aspirate revealing a cluster of malignant cells (M) of a squamous cell carcinoma on a background of normal lymphocytes and erythrocytes. Scale bars: a-d, f-h) $20 \mu \mathrm{m}$; el $100 \mu \mathrm{m}$.

\section{Discussion}

We report the first in-human, endoscopic, real-time study to identify lung cancer using nCLE. With nCLE, malignant cells could be identified in a real-time fashion in the vast majority (90\%) of patients with lung cancer. Multiple blinded raters could consistently interpret nCLE imaging of malignant lung tumours and differentiate malignant from benign reactive lymph nodes after a short training session. The outcomes of this study show the potential of the nCLE technique as a real-time guidance and feedback tool to identify the optimal area for sampling/biopsy or treatment.

Besides potentially improving current diagnostic approaches, integration of nCLE in the recently developed robotic bronchoscopic navigational technology [3] may result in an optimal real-time bronchoscopic lung cancer detection tool.

Initial experience with the CLE technique was gained in lung cancer by scanning the airway mucosa and lung tumours using a pCLE approach [7,9]. However, with pCLE, individual malignant cells are not identified despite the use of a safe [15] fluorescent dye (fluorescein). This is probably due to the fact that pCLE is limited to scanning the surface of the lung tumour and the thickness of the image plane $(50 \mu \mathrm{m})$ causes overlap of cells on the imaging that leads to dark hollows.

nCLE is a novel approach that allows for imaging on a cellular level on the inside of the tumour, with an adjusted submillimetre probe with a narrower width of the image plane $(30 \mu \mathrm{m})$. In the field of

TABLE 2 Observer performance ( $n=7$ ) using the three identified nCLE criteria for the presence of lung cancer based on 34 nCLE videos from 20 patients

\begin{tabular}{lcccccc}
$\begin{array}{l}\text { Validation session } \\
\text { (total 238 ratings) }\end{array}$ & Tumours $\mathbf{n}$ & Sensitivity & Specificity & Accuracy & PPV & NPV \\
\hline Malignant tumours & 6 & 90.5 & 89.3 & 90 & 92.7 & 86.2 \\
Metastatic lymph nodes & 21 & 89.8 & 89.3 & 89.6 & 93.6 & 83.3 \\
\hline
\end{tabular}

nCLE: needle-based confocal laser endomicroscopy; PPV: positive predictive value; NPV: negative predictive value. 
TABLE 3 IOA for the proposed nCLE criteria for malignancy and for the final diagnosis between the seven raters during the first validation session

\begin{tabular}{|c|c|}
\hline & к-value \\
\hline Dark enlarged pleomorphic cells & $0.48(0.46-0.50)$ \\
\hline Dark clumps & $0.31(0.29-0.33)$ \\
\hline Directional streaming & $0.62(0.60-0.64)$ \\
\hline \multicolumn{2}{|l|}{ Final diagnosis } \\
\hline IOA & $0.68(0.66-0.70)$ \\
\hline 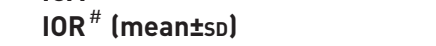 & $0.70 \pm 0.15$ \\
\hline
\end{tabular}

Data are presented as the IOA $\times(95 \% \mathrm{CI})$, unless otherwise stated. nCLE: needle-based confocal laser endomicroscopy; IOA: inter-observer agreement; IOR: intra-observer reliability. \#: comparing the first and second validation sessions for the final diagnosis. Landis-Koch interpretation system: poor $<0.2$, fair $0.21-0.4$, moderate $0.41-0.6$, substantial $0.61-0.8$ and excellent $0.81-1$.

gastero-enterology, nCLE has shown to be of additional value to conventional needle aspiration in pancreatic cancer $[16,17]$. It is supportive of our findings that studies in pancreatic adenocarcinoma describe the presence of similar malignancy features (dark enlarged pleomorphic cells and dark clumps) to those we found in lung tumours and involved mediastinal lymph nodes.

The experience of nCLE in lung cancer is currently limited to case reports. Our group has shown that it is feasible to identify tumour cells in a pulmonary tumour [10]. Another case series of 10 patients with lung and mediastinal masses could not identify individual malignant cells in CT-guided nCLE-assessed lung tumours, possibly due to technical reasons (the absence of real-time nCLE guidance) or learning curve issues [18].

The current nCLE study demonstrates that, in a group of 21 lung cancer patients, individual malignant cellular structures were present and consistently recognised in lung tumours and nodal metastases in a real-time fashion. Based on three characteristics, raters could interpret nCLE imaging with substantial agreement for the presence of malignancy, and repeat their performance (IOR). The features of "dark enlarged pleomorphic cells" and "dark clumps" were most valuable for detecting malignancy in lung cancer, featuring in $85 \%$ (22 out of 26 videos) and 38\% (10 out of 26 videos) of malignant lesions. The combination of these two features was present in $88 \%$ of the malignancy videos.

Although the agreement between the raters regarding the presence of the "directional streaming" characteristic was higher than the other two characteristics (IOA 0.62), this feature was present in only a few of the malignant cases (11\%) and adding this feature to the other two did not lead to the identification of additional malignancies.

Currently, the nCLE probe is limited to use with the 19G EUS needle because it does not fit through the single clinically available 19G EBUS needle. This is a temporary issue because other 19G EBUS needles with an increased inner diameter are in development.

A limitation of this study is the selection of the video sequences. In the same fashion as most published validation studies of CLE in other organs $[16,19]$, not all collected video material was shown to make it feasible for the seven raters to evaluate the nCLE videos in a limited amount of time.

For future lung cancer studies we recommend that the needle probe be moved slowly forwards for optimal image quality. In our experience, a backwards motion leads to obscured imaging due to the presence of erythrocytes from damaged vessels. The time to image a lymph node with nCLE over the full diameter in a single plane was $\sim 10 \mathrm{~s}$, including fanning the needle with a slightly changed angle to increase the imaged area.

The widely available and safe contrast agent fluorescein was used to obtain nCLE imaging in this study [15]. Advantages of using intravenous fluorescein are that it works rapidly $(<1 \mathrm{~min})$ and stains the extracellular matrix, which enables the shape of cellular structures and vessels to be visualised $[16,18,20]$. It should be noted, however, that nCLE in its current form cannot be used as a substitute for pathology or improve ROSE, because nCLE with fluorescein does not stain the nucleus of cells and does not allow for tumour marker analysis, which is important in determining lung cancer subtypes and the optimal treatment strategy. Currently there is no evidence that nCLE is superior to ROSE for detecting tumours in lung lesions. Prospective studies comparing the two modalities for on-site identification of tumour cells are needed. 
Pending technological improvements and confirmation of our findings, our results show the potential of nCLE as a guidance tool to identify the optimal area for sampling and as a real-time feedback technique for diagnostic, staging and treatment procedures in lung cancer. Integration of nCLE into the recently developed robotic bronchoscopic navigational technology [3] may result in an optimal real-time bronchoscopic lung cancer detection tool.

In conclusion, we showed that endosonography-guided nCLE imaging in lung cancer is feasible and safe. nCLE enabled "live cell imaging" of malignant cells and was consistently interpreted.

Acknowledgements: We thank the patients who participated in this study. We thank Marijke Twisk, Rob Hoogvorst (Dept of Pathology), Laurence Crombag and Joris Veltman (Dept of Pulmonology), Jan-Erik Freund and Esmee Liem (Dept of Urology) for their time and effort in rating the CLE videos.

Conflict of interest: J. Yared has nothing to disclose. D.M. de Bruin has nothing to disclose. S.L. Meijer has nothing to disclose. P. Baas has nothing to disclose. P.I. Bonta has nothing to disclose. J.T. Annema reports non-financial material support from Mauna Kea Technologies during the conduct of the study. L. Wijmans has nothing to disclose.

Support statement: The study was designed independently from commercial parties. CLE material was purchased for a reduced fee from Mauna Kea Technologies.

\section{References}

1 Global Burden of Disease Cancer Collaboration, Fitzmaurice C, Allen C, et al. global, regional, and national cancer incidence, mortality, years of life lost, years lived with disability, and disability-adjusted life-years for 32 cancer groups, 1990 to 2015: a systematic analysis for the Global Burden of Disease study. JAMA Oncol 2017; 3: 524-548.

2 Ost DE, Ernst A, Lei X, et al. Diagnostic yield and complications of bronchoscopy for peripheral lung lesions. Results of the AQuIRE Registry. Am J Respir Crit Care Med 2016; 193: 68-77.

3 Swaney PJ, Mahoney AW, Hartley BI, et al. Toward transoral peripheral lung access: combining continuum robots and steerable needles. J Med Robot Res 2017; 2: 1750001.

4 Benias PC, D'Souza LS, Papafragkakis H, et al. Needle-based confocal endomicroscopy for evaluation of malignant lymph nodes - a feasibility study. Endoscopy 2016; 48: 923-928.

5 Giovannini M. Needle-based confocal laser endomicroscopy. Endosc Ultrasound 2015; 4: 284-288

6 Krishna SG, Brugge WR, Dewitt JM, et al. Needle-based confocal laser endomicroscopy for the diagnosis of pancreatic cystic lesions: an international external interobserver and intraobserver study (with videos). Gastrointest Endosc 2017; 86: 644-654.

7 Fuchs FS, Zirlik S, Hildner K, et al. Fluorescein-aided confocal laser endomicroscopy of the lung. Respiration 2011; 81: $32-38$.

8 Sorokina A, Danilevskaya O, Averyanov A, et al. Comparative study of ex vivo probe-based confocal laser endomicroscopy and light microscopy in lung cancer diagnostics. Respirology 2014; 19: 907-913.

9 Wellikoff AS, Holladay RC, Downie GH, et al. Comparison of in vivo probe-based confocal laser endomicroscopy with histopathology in lung cancer: a move toward optical biopsy. Respirology 2015; 20: 967-974.

10 Wijmans L, de Bruin DM, Meijer SL, et al. Real-time optical biopsy of lung cancer. Am J Respir Crit Care Med 2016; 194: e10-e11.

11 Ha SO, Kim DY, Sohn CH, et al. Anaphylaxis caused by intravenous fluorescein: clinical characteristics and review of literature. Intern Emerg Med 2014; 9: 325-330.

12 Simons FE, Ebisawa M, Sanchez-Borges M, et al. 2015 update of the evidence base: World Allergy Organization anaphylaxis guidelines. World Allergy Organ J 2015; 8: 32.

13 Landis JR, Koch GG. The measurement of observer agreement for categorical data. Biometrics 1977; 33: 159-174.

14 Fleiss JL. Measuring nominal scale agreement among many raters. Psychol Bull 1971; 76: 378-382.

15 Wallace MB, Meining A, Canto MI, et al. The safety of intravenous fluorescein for confocal laser endomicroscopy in the gastrointestinal tract. Aliment Pharmacol Ther 2010; 31: 548-552.

16 Giovannini M, Caillol F, Monges G, et al. Endoscopic ultrasound-guided needle-based confocal laser endomicroscopy in solid pancreatic masses. Endoscopy 2016; 48: 892-898.

17 Kongkam P, Pittayanon R, Sampatanukul P, et al. Endoscopic ultrasound-guided needle-based confocal laser endomicroscopy for diagnosis of solid pancreatic lesions (ENES): a pilot study. Endosc Int Open 2016; 4: E17-E23.

18 Shulimzon TR, Lieberman S. Feasibility of confocal laser microscopy in CT-guided needle biopsy of pulmonary and mediastinal tumors: a proof-of-concept pilot study. J Vasc Interv Radiol 2016; 27: 275-280.

19 Napoleon B, Lemaistre AI, Pujol B, et al. In vivo characterization of pancreatic cystic lesions by needle-based confocal laser endomicroscopy (nCLE): proposition of a comprehensive nCLE classification confirmed by an external retrospective evaluation. Surg Endosc 2016; 30: 2603-2612.

20 Polglase AL, McLaren WJ, Skinner SA, et al. A fluorescence confocal endomicroscope for in vivo microscopy of the upper- and the lower-GI tract. Gastrointest Endosc 2005; 62: 686-695. 